\title{
Carnets
}

Revue électronique d'études françaises de l'APEF

Deuxième série - 12 | 2018

Théorie Mimétique et Études Littéraires

\section{Le paradigme du bouc émissaire chez Maupassant : une démythification narrative}

Notes sur Pierre et Jean et Boule de Suif

\section{Diane Aymard Obono Oyono}

\section{(2) OpenEdition}

\section{Journals}

Édition électronique

URL : http://journals.openedition.org/carnets/2441

DOI : $10.4000 /$ carnets. 2441

ISSN : 1646-7698

Éditeur

APEF

Référence électronique

Diane Aymard Obono Oyono, «Le paradigme du bouc émissaire chez Maupassant : une

démythification narrative », Carnets [En ligne], Deuxième série - 12 | 2018, mis en ligne le 29 janvier 2018, consulté le 03 mai 2019. URL : http://journals.openedition.org/carnets/2441 ; DOI : 10.4000/ carnets. 2441

Ce document a été généré automatiquement le 3 mai 2019.

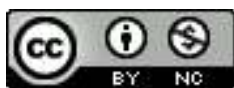

Carnets est mis à disposition selon les termes de la licence Creative Commons - Atribution - Pas d'utilisation commerciale 4.0 International. 


\section{Le paradigme du bouc émissaire chez Maupassant : une démythification narrative}

Notes sur Pierre et Jean et Boule de Suif

\section{Diane Aymard Obono Oyono}

1 L'expression "bouc émissaire», généralement employée au sens figuré aujourd'hui, renvoie à une personne sur laquelle on fait retomber les torts des autres. Le bouc émissaire est le synonyme approximatif du souffre-douleur. Il trouve son origine dans la Bible, au livre de Lévitique 16, où on peut lire que le prêtre d'Israël posait ses deux mains sur la tête d'un bouc. De cette manière, on pensait que tous les péchés commis par les Juifs étaient transmis à cet animal. Ce dernier était ensuite chassé au désert d'Azazel pour servir d'émissaire et y perdre tous les péchés.

2 S'il est bien vrai, que les hommes n'ont plus recours aux sacrifices rituels, il existe cependant toujours des boucs émissaires voilés en termes d'individus ou de groupes sociaux.

3 La littérature, tout comme l'anthropologie ou l'ethnocritique, rend compte de ces changements moraux et psychologiques. Elle réécrit, dénonce, ou rappelle l'histoire de ces boucs émissaires ignorés et oubliés ${ }^{1}$, mettant en exergue de nouvelles visions du monde. Un monde toujours empreint de violence et de rivalité entre les hommes. Cette particularité de la littérature tient au fait qu'elle est un art fortement marqué par l'écriture et la narration en prose, dont le roman et la nouvelle constituent les parangons. En effet, par la description et le récit, un auteur peut laisser lire la manifestation d'une forme de violence psychologique ou sociale d'un groupe d'individus à l'endroit d'un tiers, diffèrent ou gênant. Un lecteur averti peut ainsi être amené à y rechercher l'expression d'un processus victimaire en plein essor. Ce qui est perceptible dans les textes de Maupassant dont la particularité est d'aborder des thématiques en rapport avec des personnages marginaux, en conflit avec la société. Pierre et Jean, « récit d'adultère » et premier roman de Maupassant traite de la rivalité entre deux frères, avant de se décliner peu à peu sur l'aspect de névrose psychologique dont fait montre Pierre, au long du récit. 
La nouvelle Boule de Suif, parue en 1880 aux Soirées de Médan, est de celles qui ont fait la célébrité de notre auteur. Récit de guerre, elle met en scène un personnage à priori sans vertu face à une société microscopiquement caricaturée, « entachant [ainsi] l'honorabilité de certaines classes sociales » (Botterel-Michel, $2000: 62$ ). On peut lire au fil des textes la situation opposant l'un à sa famille d'une part et le comportement patriotique, voire héroïque, de l'autre, aux caractéristiques antithétiques, s'opposant à l'attitude lâche et égoïste de ses supposés camarades de diligence d'autre part. Ces personnages éponymes s'inscrivent dans ce schéma de personnages de type maupassantien : l'enfant illégitime, le névrosé en proie à la démence, la femme adultérine et la prostituée, etc. Reflets d'un siècle, d'une société, ils constituent la portée symbolique d'un imaginaire ${ }^{2}$.

Formulée comme suit: "Le paradigme du bouc émissaire chez Maupassant: une démythification narrative. Notes sur Pierre et Jean et Boule de Suif ", cette étude suscite toutefois quelques questionnements en ce qui concerne les convergences décelables entre la théorie du bouc émissaire et sa lisibilité dans les textes maupassantiens. L'un rend-t-il compte des travaux de l'autre? Ou les deux (théoricien et écrivain) s'inscrivent-ils juste dans une sorte de complémentarité paradigmatique? Nous savons en effet que le texte littéraire constitue un matériau inestimable, en ce qui concerne la construction de certaines théories développées dans les sciences humaines. Sa lecture permet avant tout de produire un système de pensées critique relatif à la compréhension de la société et de l'existence humaine. Ainsi, quels procédés nous permettraient à cet effet, de penser la fonction démythificatrice de Maupassant dans ces textes? Enfin, peut-on parler de paradigme ou des paradigmes du bouc émissaire?

La notion de paradigme désigne ce que les membres d'une communauté scientifique donnée possèdent en commun, c'est-à-dire l'ensemble des techniques, des modèles et des valeurs auxquels les membres d'une communauté adhèrent plus ou moins consciemment ${ }^{3}$ . Partant de son sens biblique, il sera question de noter la résurgence de ce phénomène dans les sociétés archaïques et celles dites modernes. Notre entreprise heuristique consistera ensuite, non seulement à identifier les boucs émissaires de notre corpus (Pierre et Boule de Suif) par l'entremise des stéréotypes victimaires à l'œuvre, mais aussi à démontrer comment la structuration dudit mécanisme se donne à lire pour enfin en déduire la preuve d'une démythification réelle qu'en fait Maupassant, par ses textes.

\section{I - Le bouc émissaire : de la Bible à René Girard}

6 D'origine religieuse, la notion de bouc émissaire est employée pour la première fois dans le «textus vulgatus» (Jacques, 1982). Cette traduction de « caper emissarius» n'apparait pas dans la Bible, mais vient du latin ecclésiastique d'une traduction de la Vulgate. Elle exprime ainsi en partie des concepts exogènes à la tradition juive.

Dans le livre de Lévitique16, le bouc émissaire a pour origine la dénomination animale qui renvoie à ce bouc choisi «pour faire sur lui le rite de l'absolution des péchés en l'envoyant à Azazel au désert » (Bible de Jérusalem, [1988], 2004).

La notion de bouc émissaire, de son étymologie latine "emittere ", " envoyer de hors", qui a donné les termes " emisarius ", " agent, émissaire » et " emissarium ", " déversoir ", désigne non seulement cet animal, victime expiatoire, mais aussi le rituel dont il fait l'objet. Le rituel du bouc émissaire, ou encore d'expiation se pratique généralement dans un lieu particulier « un sanctuaire » (Lv.16, 3) et s'accompagne d'un cérémonial, à l'instar 
d'une "tunique sacrée» (Lv.16, 4) et de deux boucs offerts en sacrifice. Le sang du premier bouc servira à la purification du temple. Pour le second, Aaron poursuit le rite et « impose les deux mains sur la tête du bouc vivant : il confesse sur lui toutes les fautes des fils d'Israël et toutes leurs révoltes, et il les met sur la tête du bouc, puis il l'envoie au désert». C'est le rite d'expiation du bouc d'Azazel tel que décrit par le judaïsme. Ce dernier bouc est représentatif du sens contemporain du bouc émissaire, celui sur qui l'on effectue un transfert de culpabilité, pour être en phase avec notre conscience. Il incarne tout ce qu'il y a de mal en l'homme, du moins tout ce que ce dernier estime être différent, nocif...

9 Au fil du temps et des répétitions incessantes et inconscientes du processus victimaire, le sens anthropologique de cette expression s'est vu connoté à celui des « rites analogues à celui du Lévitique dans toutes les communautés humaines » (Girard, 2005 : 165). Il s'agit de rites à caractère religieux qui ont pour finalité le sacrifice d'une victime, dans l'espoir de "se défaire des désordres et des violences à l'intérieur de ces communautés, par l'intermédiaire de victimes animales ou humaines violemment expulsées ou massacrées » (Girard, 2005 : 165).

10 Le rituel du bouc émissaire tel que défini par René Girard obéit à une séquence narrative assez précise et visible dans les textes de Maupassant, s'appuyant sur une situation de crise donnée dont l'issue se trouve dans la résolution du problème, notamment par le sacrifice ou l'expulsion d'un bouc émissaire préalablement choisi. Ce nouveau paradigme du bouc émissaire l'identifie comme « celui sur qui on fait retomber les responsabilités ". Le regard qu'on lui porte en fait en quelque sorte, un souffre-douleur. Il est perçu différemment, reconnu comme étant, bien souvent, une "victime innocente ». Ces boucs émissaires sont généralement ceux sur qui on cristallise sa frustration et son mal-être. Du point de vue de notre théoricien, le rituel victimaire possède désormais un sens psychosocial qui « apparait au grand jour dans notre société parce qu'il n'est pas ritualisé, [mais demeure une] tendance, universelle chez les hommes, à décharger leur violence accumulée sur un substitut, sur une victime de recharge » (Girard, $2001: 29$ ).

\section{II - Les figures du bouc émissaire moderne}

11 Dans son ouvrage, Le Bouc émissaire, René Girard met en avant les hypothèses reliant le thème à son mécanisme perceptible dans des textes médiévaux dont le plus illustratif est celui de Guillaume Machaut, Le Jugement du roi de Navarre. Il nous fait d'ailleurs constater que ce type de texte appartient à la rubrique des textes dits de persécution où l'on fait généralement le procès des sorcières, des registres de l'inquisition, etc. Les persécutions qu'y subissent les victimes de ces violences accusées injustement sont dites légales, mais également encouragées par une opinion publique surexcitée : c'est la logique de la foule. Ces textes comprennent souvent une dimension imaginaire et fantastique. Toutefois, une lecture attentive à la présence de certains « stéréotypes " permet de "repérer les traces de persécutions bien réelles» (Girard, 1972 : 83). Pour Maupassant, face à la foule, il y a ces personnages souvent victimes de leur apparence au point de perdre toute notion de leur véritable identité, « l'individu est alors confronté à une dichotomie entre son Moi et le reflet de celui-ci qu'il offre aux autres » (Botterel-Michel, $2000: 380$ ).

L'un roman d'adultère et l'autre nouvelle de guerre, ces textes mettent par leurs caractéristiques, l'accent sur la question du désir triangulaire (Pierre-Mme RosemillyJean, Marechal-Mme Roland-M.Roland), de la crise mimétique et de la violence. 

une plus intime. Souvent antithétiques, ils s'érigent de par leurs attributs physiques et psychiques en des personnages marginaux. Ce sont des prostituées au grand cœur, des êtres terriblement seuls et souvent angoissés, des enfants illégitimes, des âmes romantiques; ou des "âmes malades ", etc. Autant de critères qui déterminent par la suite le rôle d'aliénés qu'ils joueront au fil du texte. Ces éléments permettent de les identifier facilement. Car nous dit René Girard :

À côté des critères culturels et religieux, il y en a de purement physiques. La maladie, la folie, les difformités génétiques, les mutilations accidentelles et même les infirmités en général tendent à polariser les persécuteurs. (...) Tout individu qui éprouve des difficultés d'adaptation, l'étranger, (...), le fauché, ou tout simplement le dernier arrivé, est plus ou moins interchangeable avec l'infirme. (Girard, 1972: 29)

L'aspect fatalitaire de leur destin semble avoir pour prédicat le regard de l'autre, donc de la société sur eux. Boule de Suif, aux yeux de la société, fait partie des "déclassés », des invalides de la société française, de par son statut de prostituée et de femme. En effet, dans le texte, aussitôt identifiée, en dépit de son charme, c'est avant tout son statut d'« une de celles appelées galantes » (Maupassant, 1974: 91) qui est mis en avant par ses voisins de diligence et même par le narrateur. Pour ses compagnons de route, son statut sémantique, se résumerait en termes de : "prostituée »; " honte publique »; " vendue sans vergogne » (Maupassant, 1974 : 92). Les signes victimaires ou « signalement » d'elle en font un potentiel bouc émissaire en situation de crise. L'auteur insiste toutefois en précisant, comme pour lui ôter une épine du pied, ou pour mieux l'enfoncer, qu'en plus d'être une prostituée, « elle était bonapartiste ». Notons cette particularité du narrateur à toujours mettre en éveil le lecteur dans la description qu'il fait d'un personnage. Force est de constater que :

Le romancier transforme ainsi les valeurs de la société qui ont pour habitude d'être établies comme normes. Il dote les êtres de traits inattendus et «anormaux». Le prêtre de Maupassant peut être aussi cruel, la prostituée d'habitude vénale se montre patriote (...) l'écrivain met l'accent sur le travestissement de la vérité, sur la dichotomie entre l'être et le paraitre. (Botterel-Michel, 2000 : 62)

Nombreux seront ainsi ces personnages à double facette qui se verront persécutés pour ce qu'ils paraissent. Boule de Suif se constituera en une victime de l'égoïsme et de la lâcheté de ses compagnons de route, tout comme le statut de «bâtard» fera défaut au petit Simon dans «Le papa de Simon », et lui vaudra de se faire lyncher par ses camarades de classe.

Les déterminations extérieures paraissent donc sceller le destin de nombreux personnages mis en scène par Maupassant: difformités physiques, filiation illégitime, etc.

D'autres encore sont victimes d'un mal plus profond, fatalité plus intime en rapport avec l'âme. Nombreux sont ces personnages de Maupassant en proie à la psychose suite à une douleur émotionnelle, voire sentimentale qui détruit en eux toute possibilité d'espoir: Comment espérer quand tout s'écoule et se dissout autour de nous? Quand tout est sujet à une situation de crise : crise de l'amour, crise des relations sociales, crise des valeurs ? D'où la résurgence de la notion de pessimisme chez les personnages de type maupassantien. En effet, Pierre fera l'expérience de la déception et de la désillusion, suite à la découverte de l'infidélité de sa mère. En personnage blessé, « il comprenait à présent que, l'aimant, il ne l'avait jamais regardée » (Maupassant, 1987: 17), il n'avait jamais 
perçu sa mère comme un être humain capable de faiblesse, mais plutôt semblable à un dieu, un modèle qui ne saurait déshonorer l'image de sa famille. Il découvre à ses dépens, la loi bourgeoise de l'amour et du mariage.

18 La rivalité et les réflexions obsessionnelles conduisent à le plonger dans la démence et la perte d'identité. Par la récurrence des monologues, nous pouvons percevoir un personnage qui souffre au fond de solitude et par extension d'incompréhension. André Vial nous fait d'ailleurs remarquer la particularité de certains personnages féminins à l'instar de Jeanne dans Une Vie, qui se donne à voir comme un personnage "bovarique ». Il nous dit en effet que Maupassant fait une interprétation "bovarique » de l'univers en démontrant par ses boucs émissaires « l'éternelle misère de tout ». Ainsi, Une Vie, ou la plupart des textes maupassantiens, mettent en scène des femmes bourgeoises malmariées et font montre d'une dénonciation notoire d'une forme d'éducation féminine favorable au spleen (Vial, 1994 : 111). Pierre est le témoin et la victime de cette triste réalité. Il se trouve dès lors chargé d'une contre-identité, d'une paratopie ${ }^{4}$ (Maingueneau, 2004) spatiale et temporelle mêlée d'une vision négative de la société bourgeoise. Il croyait appartenir à une famille, à un milieu, à une société avec des valeurs et des réalités propres et immuables auxquelles il croyait. Soudain se fait le réveil qui le fait passer d'un état d'appartenance (sa famille, père, mère et frère, la classe bourgeoise et ses valeurs) à un état de non-appartenance, dans la mesure où il ne se reconnait pas dans cette manière de vivre qui lui semble une trahison. Cette perversion de la normalité que transcrit le narrateur est ce qui prédispose le personnage à un lynchage collectif : il ne s'identifie pas aux autres, il n'accepte pas l'infamie, il refuse une quelconque appartenance à ce milieu, à cette famille aux valeurs illusoires. Il se trouve de ce fait perçu dans ce texte comme " celui par qui le scandale arrive " préfigurant ainsi son expulsion d'un univers où il ne trouve plus sa place. De même que Paul dans «La femme de Paul », victime de l'infidélité de sa maitresse qui se révèle être bisexuelle, sera mené au suicide à la suite de cette découverte, laissant cette dernière dans les bras de son amante. Il s'agit souvent de personnages assez idéalistes voire romantiques, des "âmes sensibles ». Maupassant comme Flaubert créent ainsi un personnage type, romanesque, qui se trouve dans l'incapacité de supporter la destinée qui lui incombe pour avoir trop idéalisé cette réalité » (Botterel-Michel, $2000: 43$ ).

Le bouc moderne dans ce contexte qui est le nôtre se définit par sa particularité extérieure telle que la prostitution pour Boule de Suif, mais aussi sur la base de déterminants psychologiques tels que la colère, la jalousie, voire la violence pour Pierre. Saad Gaddar nous rappelle que :

En effet, le monde était conçu par les naturalistes comme un modèle biologique où

il faut repérer la pathologie sociale pour la dénoncer et cette dénonciation a été faite au moyen d'une focalisation et un intérêt pour les « cas limites » incarnés par des personnages marginaux. (Gaddar, $1998: 38$ )

Dans un siècle bourgeois où tout semble être à sa place, « suivant l'usage qu'on en fait » et qui a pour principes : le Beau, le Vrai et le Bon, la présence de la prostituée fait tâche face à des personnages représentatifs d'une époque, microcosme d'une société: la classe bourgeoise, le clergé et les démocrates. À cela s'ajoute le caractère révolté de Pierre face à son frère illégitime et à sa mère infidèle. Autant de critères qui constituent des signaux d'alarmes à l'application du mécanisme émissaire. 


\section{III - Construction et déconstruction du bouc émissaire}

\section{De la crise à l'expulsion}

21 La crise est le véritable point de départ de la trame narrative de ces textes : crise de la société contemporaine par brouillage des classes sociales et de leur rôle, crise de la foi, crise de l'identité, qu'elle soit identité sexuelle ou identité du Moi » (Botterel- Michel, 2000 : 5). De façon globalisante, le mécanisme du bouc émissaire fonctionnerait selon les temps suivants : a) une société aux prises avec de graves difficultés qui mettent en péril son existence même, car elles voient nécessairement leur répondant dans des conflits internes, suivis de représailles indéfinies, sans que jamais puisse s'arrêter le cercle vicieux de la violence réciproque ; b) sans raisons bien définissables, la violence interne du groupe se polarise sur un individu qui se trouve désigné comme responsable de tous les maux dont souffre le groupe ; c) une sorte de miracle se produit alors, le groupe fait son unité et retrouve par conséquent la paix intérieure qui lui faisait défaut par le sacrifice d'un bouc émissaire; d) rétrospectivement, chacun se conforte dans la certitude que l' individu mis à mort était bien responsable des maux de la société. Le groupe éprouve donc paradoxalement de la gratitude envers celui par qui la paix est revenue. Ainsi détaillé, il s'appuie sur un mode de séquence narrative-type, perceptible dans Pierre et Jean et Boule de Suif.

Nous le savons, c'est souvent dans une société traversée par une crise qu'on recourt à la victimisation. René Girard souligne que :

Fréquemment, ce qui trouble la paix, c'est une épidémie mal définie (...) Il peut s'agir aussi d'une interruption de fonctions vitales causée par une espèce de blocage, de paralysie. Il peut s'agir aussi des désastres plus ordinaires, de famines d'inondations, de sécheresses destructrices et autres catastrophes naturelles. Toujours et partout on peut résumer la situation initiale en termes d'une crise qui fait peser sur la communauté et son système culturel une menace de destruction totale. (Girard, [1999], $2001: 89$ )

Pierre et Jean, rappelons-le, est le récit d'une déchirure familiale. En effet, trois situations de crise sont à relever au cours desquelles les frères se trouvent confrontés à une configuration de mimétisme triangulaire, notamment: Pierre-Jean-Madame Rosemily ; Pierre-Jean-Appartement ; Pierre-Jean-Madame Roland. Ils représentent le schéma sujetdésirant-médiateur-objet désiré. La mimétique triangulaire qui nous intéresse dans ce texte est celle qui unit Pierre-Jean-Madame Roland. Ce triangle sera principalement à l'origine de la crise chez les Roland. Car c'est particulièrement l'annonce du legs à Jean plutôt qu'aux deux autres qui plongera cette famille dans une spirale victimaire, dont le retour à la paix ne sera possible que par l'expulsion de Pierre.

Boule de Suif s'inscrit dans un contexte de guerre franco-prussienne [1870]. La crise a lieu en Normandie dans une diligence et se poursuit dans une auberge. Boule de Suif et ses voisins sont en fuite vers le Havre quand un officier Prussien les empêche de continuer leur voyage : «Je ne veux pas qu'ils partent sans mon ordre » (Maupassant, $1974: 104)$. Le personnage éponyme se trouve sollicité par le soldat Prussien pour assouvir la libido de celui-ci, sans quoi la diligence ne reprendrait pas sa route. Ce qui ne sera possible cette fois que par le sacrifice de la prostituée. l'indifférenciation. En effet, c'est au fil des chapitres que se construit l'image de la victime 
émissaire du personnage de Pierre, allant de la rivalité mimétique (chap. I ; II ; III) à l'indifférentiation et à la violence qu'il manifeste suite à la confirmation de ses doutes sur l'adultère de sa mère. La crise mimétique que subit la famille conduit à l'indifférenciation. Elle se caractérise par une situation de perte de valeur, de rupture du contrat social. Le non-respect de sa mère vis-à-vis de ses engagements maritaux conduit à une déchirure du cœur de Pierre qui frôle peu à peu la folie avec « des phrases d'halluciné ». Le lien familial devenu un «mensonge filial », «la main d'un mort» avait frappé sa famille. Un passage révèle particulièrement cette crise de l'indifférence que connait Pierre: «Sa famille! Depuis deux jours une main inconnue et malfaisante, la main d'un mort, avait arraché et cassé, un à un, tous les liens qui tenaient l'un et l'autre ces quatre êtres. C'était fini, c'était brisé...» (Maupassant, 1987 : 146). C'est la digue qui canalisait les rapports de force réels qui vole en éclats, laissant libre cours à la violence et à la destruction (Ndong, 2012). Face au refus du soldat Prussien, l'indifférenciation est le fait de la situation de stress et de confusions que traversent les compagnons de Boule de Suif. Ils n'ont pas la paix, « une fois rentrés, on ne sut plus que faire. Des paroles aigres furent échangées à propos de choses insignifiantes. Le diner dura peu, et chacun, espérant dormir pour tuer le temps » (Maupassant, 1974 : 109).

À l'indifférenciation succède la polarisation de la violence " tous contre un », sur celui qui attire «l'attention haineuse et coléreuse des membres de la communauté" (Ndong, 2012: 177). Pierre est cette victime émissaire qui choisit elle-même d'être expulsée de sa communauté, de sa famille. En effet, la conduite désagréable de Pierre à l'endroit de sa mère menace de plus en plus l'équilibre familial paisible auparavant. C'est donc dans un commun accord que la mère adultérine et son fils illégitime choisissent d'éloigner ce perturbateur meurtri qu'est Pierre. Clergé, bourgeoisie et démocrates choisissent de faire alliance en vue de sortir de ce cycle de violence réciproque : «Alors on conspira » (Maupassant, $19874: 111)$; " puisque c'est son métier à cette gueuse ", « il faudrait la décider ", « on prépara le blocus comme pour une forteresse investie ». Face à « la logique de la foule » (Girard, $1982: 21$ ), ils deviennent tous deux :

Le monstre (qui) hérite de tout ce qu'il y a de détestable dans l'affaire, la crise, les crimes, les critères de sélection victimaire, les trois premiers stéréotypes persécuteurs. Le héros (Jean, les compagnons de diligence) incarne le quatrième stéréotype seulement, le meurtre, la décision sacrificielle, d'autant plus ouvertement libératrice que la méchanceté du monstre en justifie pleinement la violence. (Girard, 2009 : 190)

L'annonce du crime rapproche les bourreaux qui, voyant leur environnement menacé, optent en guise de solution pour la mise hors d'état de nuire de ces éléments gênants et perturbateurs que représentent Pierre et Boule de Suif. La phrase d'appel au secours de Madame Roland à l'endroit de Jean l'atteste : «Sauve-moi de lui, toi, mon petit, sauvemoi, fais quelque chose (...) j'ai si peur de lui... Si peur !» (Maupassant, 19874 : 190). Elle lui permet de se déculpabiliser et d'effectuer ainsi un transfert de sa culpabilité sur Pierre symbole de monstruosité et de violence. La colère de Pierre l'aveugle et aveugle ses persécuteurs qui voient en lui un réel danger. Retenons que le choix d'expulser Pierre ou de livrer Boule de Suif au Prussien n'est pas le fruit d'un hasard, mais découle d'une mise en exergue des signes victimaires de l'un et de l'autre. Parce que «la perfection du bouc émissaire c'est son unanimité » (Girard, 1982: 52), Pierre incarne le mal-aimé, rejeté tour à tour par Madame Rosemily, puis sa mère dont la trahison rend répugnante et qui, avec son frère Jean, choisissent de le sacrifier pour leur plus grand bien. Boule de Suif incarne à la fois la prostituée et la femme dans la société française, elle est destinée à jouer un 
rôle secondaire en plus d'être une " déclassée ». Tout comme un objet, un médiateur, elle a servi à l'assouvissement des besoins primaires de la société (nourriture et sexe).

La dernière étape de ce processus victimaire est l'expulsion de Pierre et le sacrifice de Boule de Suif. En effet, la crise mimétique débouche sur le sacrifice du bouc émissaire condamné à l'errance comme le bouc d'Azazel et donc à une certaine mort de celui-ci. Toutefois, le narrateur nous montre que le choix de se sacrifier incombe aussi bien au bouc émissaire qu'à ses bourreaux. Bien que subissant une forme de violence psychologique, ces personnages choisissent de se sacrifier, de se faire expulser pour le bien de la communauté. Pierre finira expulsé de sa famille et même du texte. Boule de Suif se verra à nouveau rejetée par ceux qui lui ont semblé être ses amis : «Elle se sentait noyée dans le mépris de ces gredins honnêtes qui l'avaient sacrifiée d'abord, rejetée ensuite, comme une chose malpropre et inutile » (Maupassant, 1974 : 119).

La séquence narrative du rituel du bouc émissaire correspond à sa conception psychosociale. Chacun de ces personnages semble la proie de la manifestation d'une forme de violence ${ }^{5}$ sociale, mais aussi psychologique : ils subissent ou se voient infliger par l'Autre un préjudice moral et/ou physique.

\section{Déconstruction et démythification narratives}

En fin psychologue et surtout en "sociologue de la mondanité » qu'il décrit de façon réaliste et souvent caricaturale, c'est par « l'analyse, l'ironie, et la mise à distance » que Maupassant démystifie le rituel victimaire. Il oriente la réflexion vers la victime en révélant les manipulations des bourreaux. Le personnage qui, au départ, semblait être le bourreau à l'instar de Pierre, se découvre peu à peu n'être que la victime de fauxsemblants de ses proches, de la foule, de la société. C'est finalement, la solitude du bouc émissaire qu'il veut dévoiler. Rejeté ou expulsé, chaque personnage se voit à la fin du texte noyé dans une solitude des plus totales. Le pire châtiment qui puisse donc être infligé à un bouc émissaire n'est donc pas son sacrifice qui, selon René Girard, aboutit à la déification du sacrifié. Ce n'est pas la déification du sacrifié, à l'issue de son sacrifice que nous donne à lire l'auteur, bien que les bourreaux semblent certes reconnaissants à l'endroit de celui par qui paradoxalement, la paix est revenue, mais c'est le visage de ce dernier condamné à la solitude que nous révèle le narrateur. Pierre est condamné à vivre dans une cabine qui ressemble à s'y méprendre à un cercueil. Boule de Suif se voit mise à l'écart, délaissée et à nouveau déclassée. Pour chacun la solitude est totale.

31 L'écrivain a choisi de dévoiler le monde et singulièrement l'homme aux autres hommes pour que ceux-ci prennent en face l'objet qui aussi mit à nu leur entière responsabilité (Sartre, [1948], 2008). Il s'insurge contre la mauvaise foi, l'égoïsme de la foule sacrificatrice. Il le signifiait déjà dans son article « Le fond du cœur » :

Je dis que le seul mobile de nos faits toujours appréciable, toujours possible à retrouver sous les guirlandes de beaux sentiments, est l'égoïsme. En effet, est-ce que tout ne se rapporte pas au moi, soit directement, soit indirectement? Toute action humaine est une manifestation d'égoïsme déguisée. (Maupassant [14 octobre 1884], $1973: 28$ )

32 De même que le christianisme (dans le Nouveau Testament), en dévoilant le sacrifice du Christ comme victime consentante, "démystifie le religieux " (Girard, 2007: 191), les textes maupassantiens démystifient le mécanisme du bouc émissaire en le rendant perceptible et conscient. Ces paroles de Cornudet à la foule sacrificatrice le démontrent : 
«Je vous dis à tous que vous venez de faire une infamie!» (Maupassant, 1974: 116). L'Autre que l'on sacrifie devient notre semblable. Pour convenir avec André Vial, nous pouvons nous permettre de penser que l'œil de Maupassant nous transcrit ce qu'il voit, mais surtout comment il le voit (Vial, 1994 : 115). Il raconte la psychologie du monde qu'il observe. Il le caricature par la stéréotypie.

La victimisation d'un personnage passe aussi par l'idée qu'il peut se faire de lui et des autres, par ses pensées comme le démontrent les monologues de Pierre. Personnage à la sensibilité " exacerbée », Pierre est à l'image de l'homme du XIXème affecté par un mal plus profond, un mal de vivre: le mal fin de siècle. N'oublions pas, comme le souligne Catherine Botterel-Michel, que « Maupassant était d'un siècle profondément pessimiste en butte à la perte des valeurs traditionnelles» (Botterel-Michel, 2000: 45). Ce qu'il déplore au plus haut point en dénonçant ici l'adultère de Madame Roland et l'attitude égocentrique qu'affichent les autres personnages à l'endroit de Boule de Suif. Il décrit le «paysage intérieur» (Vial : 1994) de ses personnages. René Girard nous fait remarquer que :

Les écrivains ont tous une manière différente d'aborder le mécanisme mimétique. Chacun appartient à une histoire, à la fois collective et individuelle. Le nombre de combinaisons mimétiques est infini, comme la façon de les exprimer. Impossible, donc, de généraliser la manière dont la mimésis fonctionne avec les écrivains. Chacun exige une démonstration entièrement différente, même si le chercheur intéressé par le mécanisme sait qu'à la fin chacun dévoilera les mêmes principes mimétiques. (Girard, $2004: 228$ ).

Les écrivains ont tous une manière différente d'aborder le mécanisme mimétique. Chacun appartient à une histoire, à la fois collective et individuelle. Le nombre de combinaisons mimétiques est infini, comme la façon de les exprimer. Impossible, donc, de généraliser la manière dont la mimésis fonctionne avec les écrivains. Chacun exige une démonstration entièrement différente, même si le chercheur intéressé par le mécanisme sait qu'à la fin chacun dévoilera les mêmes principes mimétiques. (Girard, $2004: 228$ ).

Placée sous le signe du désenchantement, cette étude raconte l'histoire ou la perte de l'innocence et des illusions de nos héros de façon systématique, en les mettant face à la réalité des relations humaines. Maupassant se fait, comme le souligne Marie Claire Bancquart, «l'un des interprètes les plus éloquents et les plus touchants (...) de la crise que traversait son époque tout entière " (Bancquart, [1976], 2002 : 17). Il met en lumière deux notions chères à René Girard, celles de la violence (symbolique et collective) et du sacrifice (s'agissant de transfert de la faute et de lynchage) modernes.

Bien qu'ancien, le mécanisme du bouc émissaire est générateur et régénérateur de ce système auquel on recourt toujours plus ou moins consciemment (Girard, 1982: 83) en situation de crise. Toutefois, il est récurrent et voilé dans nos sociétés actuelles. Au paradigme universel de ce mécanisme, tel que théorisée par René Girard, il y a la logique «spécifique » et singulière de l'exemple dans les textes de Maupassant. L'apport de la littérature demeure ainsi celui de dévoiler la dimension violente de cette théorie en laissant entrevoir les visages du bouc émissaire et leur construction, de la crise à l'expulsion.

La reconstitution du processus victimaire par nos textes démontre que seules la Bible et la littérature dénoncent véritablement les injustices faites aux victimes. Nous l'avons remarqué, «Maupassant applique à la narration un précepte pédagogique. Afin de faire agir son auditeur, il (...) passe par la provocation» (Place-Verghnes, 2005 : 148). Parce 
qu'il veut expliquer et éduquer, le choix du statut social de ses personnages fait régulièrement appel à la stéréotypie qui constitue une voie sûre d'herméneutique. Parce qu'il ne cherche pas à plaire, il dévoile ainsi un rituel inconscient pour bons nombres de consciences. De par son désir d'impersonnalité, « contrairement à d'autres, il ne bascule ni dans le mélodrame ni dans l'invraisemblance ni dans l'auto-analyse » (Lenoir, 1994 : 33).

\section{BIBLIOGRAPHIE}

BERGSON, Henri (2007). Le Rire. Paris : Presses Universitaires de France.

BINH, n.t., Viviani, Christian (1991). Lubitsch. Paris : Rivages/Cinéma.

DANTE (1956). La Divine comédie. Paris : Garnier.

EL-KHOURY, Toufic (2016). La comédie hollywoodienne classique (1929-1945). Structure triadique et médiations du désir. Paris : L'Harmattan, « Champs visuels ».

FARRUGIA, Francis (2010). « Le syndrome narratif : une 'inquiétante étrangeté'" , Sociologies [En ligne], Dossiers, Émotions et sentiments, réalité et fiction [disponible le1/6/2010] URL : http :/ sociologies.revues.org/index3152.html GIRARD, René (2004). " Un équilibre périlleux : essai d'interprétation du comique ", in La Voix méconnue du réel, une théorie des mythes archaïques et modernes. Paris : Livre de poche, «Biblio essais ».

GIRARD, René (2000). Critiques dans un souterrain. Paris : Le Livre de Poche, « Biblio essais ». GIRARD, René (2011). Géométries du désir. Paris : L'Herne.

GIRARD, René (1999). Mensonge romantique et vérité romanesque (1961). Paris : Hachette, « Pluriel ». LA ROCHEFOUCAULD, François de (1999). Maximes, réflexions, lettres. Paris : Hachette, « Pluriel ». MAURON Charles (1970). Psychocritique du genre comique. Paris : José Corti.

LEVIN, Harry (1986). « Critical Approaches to Shakespeare from 1660 to 1904 », in Stanley Wells (éd.). The Cambridge Companion to Shakespeare Studies. Cambridge: Cambridge University Press.

LEWIS, Allan (1969). « Midsummer's Night Dream, Fairy Fantasy or Erotic Nightmare? », Educational Theater Journal, 21:3, pp. 251-258.

POR, Katalin (2011). De Budapest à Hollywood, le théâtre hongrois et le cinéma hollywoodien (1930-1943). Rennes : Presses Universitaires de Rennes, « Le Spectaculaire ».

Rougemont, Denis de (2001). L'Amour et l'Occident (1939). Paris : Plon, « Bibliothèques 10/18 ». RoUgemont, Denis de (1996). Les Mythes de l'amour (1972). Paris : Albin Michel, « Espaces libres ». SARTRE, Jean-Paul (1971). L'idiot de la famille, Gustave Flaubert de 1821 à 1857, vol. 2. Paris: Gallimard, «NRF ». 
scoufos, Alice-Lyle (1981). "The 'Paradiso Terrestre' and The Testing of Love in As You Like It ", Shakespearean Studies, 14, pp. 215-227.

SHUMWAY, David (2003). Modern Love, Romance, Intimacy and the Marriage Crisis. New York: New York University Press.

STERNBERG-GREINER, Véronique (2003). Le Rire. Paris : GF-Flammarion, « Corpus », pp. 17-22.

\section{NOTES}

1. Emile Zola a écrit un article "J'accuse " lors de l'affaire Dreyfus. Il est publié dans le journal L'Aurore du 13 janvier 1898 sous forme d'une lettre ouverte au président Français Félix Faure.

2. Selon Wunenburger : «Est posé comme imaginaire ce qui ouvre sur des possibles, est doté d'une dynamique créatrice interne, d'une prégnance symbolique et d'une puissance d'adhésion au sujet. ». Par lui, l'homme crée un type de rapport particulier avec certains éléments de la nature. Il en est de même pour lui et son semblable, généralement désigné sous l'appellation de l'Autre (Wunenburger, $2003: 63$ ).

3. Giorgio Agamben, dans son ouvrage critique, Signatura rerum. Sur la méthode, s'appuie sur la généalogie de la notion de paradigme introduite par Thomas Khun en 1962 et reprise par Michel Foucault dans une perspective différente (Agamben, $2008: 11$ ).

4. La paratopie désigne selon Dominique Maingueneau, la relation paradoxale d'inclusion dans un espace social. En d'autres termes, elle fait référence au sentiment d'appartenance et/ou de non appartenance à une communauté donnée. A cet effet, Dominique Maingueneau souligne : «Toute paratopie, minimalement, dit l'appartenance et la non-appartenance, l'impossible inclusion dans une « topie ». Elle traduit la position intenable d'un sujet. »

5. La violence se définit comme: «Un acte s'exerçant avec force contre un obstacle: comportement d'une personne contre une autre qu'elle considère comme obstacle à la réalisation de son désir. Elle fait problème à la philosophie, niant la conscience et donc le pouvoir même de la philosophie. On la conçoit d'origine "purement naturelle » (Hobbes, Nietzsche) ou comme provenant d'une vie sociale mal organisée (Rousseau, Proudhon, Stirner). D'où également l'ambigüité du point de vue moral à son sujet; on la rejette comme oppression et absence de droit (J.J. Rousseau) ou bien on exalte ses vertus libératrices en la présentant comme une réponse à une violence toujours antérieure (K. Marx).» Dictionnaire de philosophie. Paris, Nathan, 1997, p. 397.

\section{RÉSUMÉS}

Le regard que l'on porte à l'autre détermine notre conception du bouc émissaire. Qu'il devienne le souffre-douleur ou la bête noire, il n'est rien d'autre qu'une solution à des difficultés sociétales par lesquelles l'injustice et la violence sont les derniers ressorts. Nous abordons l'utilité de la théorie mimétique dans les études littéraires qui est de dévoiler le mécanisme victimaire, l'existence des boucs émissaires et de déceler par cela l'idéologie de l'auteur. On rappellera les différents sens donnés à cette notion au fil des siècles, notamment le sens biblique, le sens anthropologique ou rituel de la victime émissaire, enfin le sens psycho-social ou moderne. Pierre 
et Boule de Suif illustrent le paradigme moderne du bouc émissaire. Ils vont subir les grandes étapes du mécanisme victimaire, passant de la crise mimétique à leur sacrifice. En nous permettant de percevoir le processus du bouc émissaire dans ses textes, Maupassant s'érige en démythificateur de la violence collective.

The way we look at the other determines our conception of the scapegoat. Whether it becomes the whipping boy or the pet peeve, it's nothing but a solution to societal difficulties by which injustice and violence are the last springs. In this study, we highlight the usefulness of mimetic theory in literary studies which is to unveil the victim mechanism, the existence of the scapegoats and thereby detect the author's ideology. It seems necessary to remind the different meanings given to this notion over centuries, in particular the biblical meaning, the anthropological or ritual meaning and the psycho-social or modern meaning. Pierre and Boule de Suif illustrate the modern paradigm of the scapegoat. They will undergo the great steps of the victim mechanism, moving from the mimetic crisis to their sacrifice. As a perception of the process of the scapegoat in his texts, Maupassant sets himself up as a demystifier of collective violence.

31 janvier 2018

INDEX

Mots-clés : bouc émissaire, signes victimaires, violence collective, Maupassant (Guy de), démythification

Keywords : scapegoat, victim signs, collective violence, Maupassant (Guy de), demystification

\section{AUTEUR}

\section{DIANE AYMARD OBONO OYONO}

Université de Lyon III

dianehaymard[at]yahoo.fr 